\title{
Reconciling the $2 \mathrm{TeV}$ Excesses at the LHC in a Linear Seesaw Left-Right Model
}

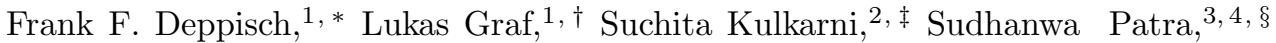

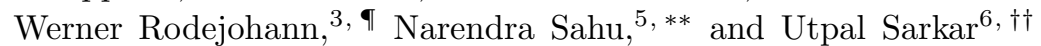 \\ ${ }^{1}$ Department of Physics and Astronomy, University College London, London WC1E 6BT, United Kingdom \\ ${ }^{2}$ Institut für Hochenergiephysik, Österreichische Akademie der Wissenschaften, Nikolsdorfer Gasse 18, A-1050 Wien, Austria \\ ${ }^{3}$ Max-Planck-Institut für Kernphysik, Saupfercheckweg 1, 69117 Heidelberg, Germany \\ ${ }^{4}$ Center of Excellence in Theoretical and Mathematical Sciences, \\ Siksha ' $O$ ' Anusandhan University, Bhubaneswar-751030, India \\ ${ }^{5}$ Department of Physics, Indian Institute of Technology, \\ Hyderabad, Yeddumailaram, 502205, Telengana, India \\ ${ }^{6}$ Physical Research Laboratory, Ahmedabad 380 009, India
}

\begin{abstract}
We interpret the $2 \mathrm{TeV}$ excesses at the LHC in a left-right symmetric model with Higgs doublets and spontaneous $D$-parity violation. The light neutrino masses are understood via a linear seesaw, suppressed by a high $D$-parity breaking scale, and the heavy neutrinos have a pseudo-Dirac character. In addition, with a suppressed right-handed gauge coupling $g_{R} / g_{L} \approx 0.6$ in an $S O(10)$ embedding, we can thereby interpret the observed eejj excess at CMS. We show that it can be reconciled with the diboson and dijet excesses within a simplified scenario based on our model. Moreover, we find that the mixing between the light and heavy neutrinos can be potentially large which would induce dominant non-standard contributions to neutrinoless double beta decay via long-range $\lambda$ and $\eta$ neutrino exchange.
\end{abstract}

PACS numbers: 98.80.Cq,14.60.Pq

\section{INTRODUCTION}

The origin of small neutrino mass, as confirmed by oscillation experiments [1, is still a mystery in particle physics. Seesaw mechanisms are the leading candidates among beyond the Standard Model (SM) scenarios to explain sub-eV masses of the neutrinos. In the conventional type-I seesaw [2], the SM is extended by three $S U(2)_{L}$ singlet right handed neutrinos $\nu_{R}$ with hypercharge $Y=0$, while in case of type-II seesaw [3] one adds a scalar triplet with hypercharge $Y=2$ to the SM. In either case, the neutrino masses can be given by $m_{\nu}=y^{2}\langle H\rangle^{2} / M$, where $M$ is the seesaw scale set by the masses of new particles and $y$ is an effective coupling. An important point to be noted in this scenario is that the new particles are $a d-h o c$ and their masses are not controlled by the SM gauge symmetry $S U(2)_{L} \times U(1)_{Y}$. Left-right symmetric models 4 , on the other hand, are extensions of the SM, where both type-I and type-II seesaw for sub-eV neutrino masses emerge naturally. The breaking of left-right symmetry then fixes the scale of seesaw $M$. By embedding the left-right symmetric model in a non-supersymmetric grand unified theory (GUT) one can find that $M$ can vary from $\mathrm{TeV}$ scale to a subGUT scale depending on the particle content and the

\footnotetext{
*Electronic address: f.deppisch@ucl.ac.uk

${ }^{\dagger}$ Electronic address: lukas.graf.14@ucl.ac.uk

‡Electronic address: suchita.kulkarni@oeaw.ac.at

$\S$ Electronic address: sudhanwa@mpi-hd.mpg.de

IElectronic address: werner.rodejohann@mpi-hd.mpg.de

** Electronic address: nsahu@iith.ac.in

${ }^{\dagger}$ Electronic address: utpal@prl.res.in
}

pattern of symmetry breaking. While the generic solution $M \approx 10^{14} \mathrm{GeV}$ and $y \approx 1$ will result in the observed small neutrino masses, models with relatively light $M \approx 100 \mathrm{GeV}-1 \mathrm{TeV}$ have the benefit that they can be probed directly at colliders such as the LHC. While this seems to require tiny couplings $y$, extending the heavy sterile neutrino sector or taking into account the flavour structure will allow the couplings and masses to conspire to produce small neutrino masses with large couplings $y$. Rather we discuss here the linear seesaw mechanism [5] and its possible implications to neutrino masses, lepton flavor violation, neutrinoless double beta decay and Collider studies in a class of $\mathrm{TeV}$ scale left-right symmetric model (LRSM).

In this paper we propose an extended left-right symmetric model (LRSM) based on the gauge group $S U(2)_{L} \times S U(2)_{R} \times U(1)_{B-L} \times D$ with doublet Higgs and spontaneous $D$-parity breaking [6], to explain the light neutrino masses via a linear seesaw mechanism. The extension of the LRSM [4] is provided by a singlet fermion $S$. We show that the suppression of neutrino mass arises through the large $D$-parity breaking scale that generates the small scale of lepton number violation, while allowing $S U(2)_{R} \times U(1)_{B-L}$ to be broken at the $\mathrm{TeV}$ scale. We embed the low energy left-right symmetric model in a non-supersymmetric $S O(10)$ GUT to calculate the difference between the left and right sector gauge couplings $g_{L}$ and $g_{R}$ after $D$-parity breaking.

The main focus of this paper is on the interpretation of the various excesses observed at the LHC around the energy scale of $2 \mathrm{TeV}$. Starting with the excess in two leptons, two jet eejj final state at CMS [7], which can be better understood in our model due to suppressed righthanded $S U(2)_{R}$ gauge coupling at the electroweak (EW) 
scale. In addition, the fact that the heavy neutrinos are of Pseudo-Dirac type guarantees that the two leptons in the process have opposite sign, in agreement with the observation (as has also been noted in Ref. 8]).

Starting with the excess in two leptons, two jet eejj final state at CMS [7, which can be better understood in our model due to suppressed right-handed $S U(2)_{R}$ gauge coupling at the electroweak (EW) scale. In addition, the fact that the heavy neutrinos are of Pseudo-Dirac type guarantees that the two leptons in the process have opposite sign, in agreement with the observation (as has also been noted in Ref. [8]).

Although the observation of the eejj excess was one of the first motivations to consider LRSMs, subsequent reports of several resonance excesses in different final states with a mass around $2 \mathrm{TeV}$ has led to an increased interest in these scenarios. Among them, most notable are, the diboson excess [9] in fully hadronic decays of the final state bosons as reported by ATLAS, the diboson excess [10] in semi-leptonic final state observed by CMS and the excess in W boson, SM Higgs final state 11. reported also by CMS. For any LRSM, explaining the eejj excess, it is important to have consistency with all these excesses as well. It is crucial to note that none of these excesses are statistically significant and the results of ongoing Run - 2 at $13 \mathrm{TeV}$ are necessary for the confirmation. We take into account the above said and few other channels to which the model under consideration is sensitive and perform a rough simultaneous fit to the mass of the heavy neutrino, the right handed $W$ boson along with their respective mixing angles.

The paper is arranged as follows. In Section II, we briefly discuss the LRSM with doublet Higgs and spontaneous $D$-parity breaking. Section III is devoted to the calculation of the neutrino mass via the linear seesaw mechanism and the consequences for neutrinoless double beta decay. Section IV estimates the mismatch between the gauge couplings by embedding the low energy LRSM in a non-supersymmetric SO(10) GUT. In Section V we then discuss how the various excesses at the LHC can be understood in our framework before we conclude in Section VI.

\section{LRSM WITH HIGGS DOUBLETS}

In the usual left-right symmetric extension 4 of the Standard Model, the gauge group is expanded to $S U(3)_{c} \times S U(2)_{L} \times S U(2)_{R} \times U(1)_{B-L} \equiv \mathcal{G}_{L R}$, where $B$ is baryon number and $L$ is lepton number. The electric charge $Q$ and the hypercharge $Y$ are related to the quantum numbers of the group as [4]

$$
Q=T_{3 L}+T_{3 R}+\frac{B-L}{2}=T_{3 L}+Y .
$$

The conservation of left-right parity implies that all lefthanded fermions have right-handed partners. Therefore, the model accommodates automatically a right-handed neutrino $N$ per family, which is singlet under the SM gauge group.

In the LRSM, the choice of the discrete left-right symmetry i.e. either Parity $\mathcal{P}$ or charge conjugation symmetry $\mathcal{C}$, plays a crucial role. In addition to the usual parity of the Lorentz group (denoted by $\mathcal{P}$ ), a discrete left-right symmetry called $D$-parity (similar to charge conjugation symmetry) that acts on the left-right symmetric gauge group is also assumed to be conserved. For the usual fermions, i.e. quarks and leptons, these two parity operations can be identified with each other. However, scalar particles, which transform under the Lorentz group trivially, can be transformed under the $D$-parity non-trivially, as they belong to non-trivial representations of the left-right symmetric group. For example, in the usual convention, the charge conjugation operator of the left-right symmetric group is identified with $D$ parity. Then the $D$-parity would transform a scalar belonging to a representation $\mathcal{R}$ of the left-right symmetric group to a representation $\mathcal{R}^{*}$. In that case the breaking of $D$-parity could take place at a different scale than the breaking of parity $\mathcal{P}$ of the Lorentz group. Since the breaking of the left-right symmetric group is always associated with the breaking of parity $\mathcal{P}$ of the Lorentz group, this means that the $D$-parity breaking scale could be decoupled from the left-right symmetry breaking scale. Details of left-right symmetric models with spontaneous $D$-parity breaking can be found in [6].

The particle content of the model and the gauge transformation under $\mathcal{G}_{L R} \times D$ is given as follows [12]. The fermionic representations are (per generation)

$$
\begin{gathered}
Q_{L}=\left(\begin{array}{l}
u_{L} \\
d_{L}
\end{array}\right) \equiv(3,2,1,1 / 3), Q_{R}=\left(\begin{array}{c}
u_{R} \\
d_{R}
\end{array}\right) \equiv(3,1,2,1 / 3), \\
\ell_{L}=\left(\begin{array}{c}
\nu_{L} \\
e_{L}
\end{array}\right) \equiv(1,2,1,-1), \ell_{R}=\left(\begin{array}{c}
N \\
e_{R}
\end{array}\right) \equiv(1,1,2,-1), \\
S \equiv(1,1,1,0) .
\end{gathered}
$$

The scalar sector consists of

$$
\begin{aligned}
H_{L} & =\left(\begin{array}{c}
h_{L}^{+} \\
h_{L}^{0}
\end{array}\right) \equiv(1,2,1,1), H_{R}=\left(\begin{array}{c}
h_{R}^{+} \\
h_{R}^{0}
\end{array}\right) \equiv(1,1,2,1), \\
\Phi & =\left(\begin{array}{cc}
\phi_{1}^{0} & \phi_{2}^{+} \\
\phi_{1}^{-} & \phi_{2}^{0}
\end{array}\right) \equiv(1,2,2,0), \quad \eta \equiv(1,1,1,0),
\end{aligned}
$$

where the quantum numbers inside the parentheses are under the gauge group $S U(3)_{c} \times S U(2)_{L} \times S U(2)_{R} \times$ $U(1)_{B-L}$. The only particle $\eta$ is odd under $D$-parity while rest of the particles are even. In the fermion sector we have included singlet fermions $S$, which will eventually couple to the left-handed neutrinos.

\section{A. Symmetry breaking}

The electroweak symmetry is broken by a bi-doublet scalar field $\Phi$, which gives Dirac masses to all charged 
fermions and also to neutrinos. In addition, we introduce a right-handed doublet scalar field $H_{R}$ and its leftright symmetric partner $H_{L}$. The former breaks the leftright symmetry and mixes the singlet fermion $S$ with the right-handed neutrinos, while the field $H_{L}$ acquires a much smaller vev and mixes $S$ with the left-handed neutrinos. Finally we add a $D$-parity odd singlet scalar field $\eta$, whose vev breaks the $D$-parity while keeping the leftright gauge symmetry intact. The symmetry breaking pattern can thus be written as 6, 12 .

$$
\begin{aligned}
S U(3)_{c} \times S U(2)_{L} & \times S U(2)_{R} \times U(1)_{B-L} \times D \\
& \downarrow\langle\eta\rangle \\
S U(3)_{c} \times S U(2)_{L} & \times S U(2)_{R} \times U(1)_{B-L} \\
\downarrow & \left\langle H_{R}\right\rangle \\
S U(3)_{c} \times & S U(2)_{L} \times U(1)_{Y} \\
& \downarrow\langle\Phi\rangle \\
S U(3)_{c} & \times U(1)_{e m} .
\end{aligned}
$$

We define the vers of the various fields as

$$
\langle\Phi\rangle=k_{1}, k_{2},\left\langle H_{L}\right\rangle=v_{L},\left\langle H_{R}\right\rangle=v_{R},\langle\eta\rangle=\eta_{P} .
$$

The scalar potential contains the usual quadratic and quartic terms. Several phenomenology aspects of this potential were previously discussed in [12]. The terms relevant for the purpose of our discussion are given by

$$
\begin{aligned}
V & \supset \mu_{h}^{2}\left(H_{L}^{\dagger} H_{L}+H_{R}^{\dagger} H_{R}\right) \\
& +\mu_{1}\left(H_{L}^{\dagger} \Phi H_{R}+H_{R}^{\dagger} \Phi^{\dagger} H_{L}\right) \\
& +\mu_{2}\left(H_{L}^{\dagger} \tilde{\Phi} H_{R}+H_{R}^{\dagger} \tilde{\Phi}^{\dagger} H_{L}\right) \\
& +M^{\prime} \eta\left(H_{L}^{\dagger} H_{L}-H_{R}^{\dagger} H_{R}\right),
\end{aligned}
$$

with $\tilde{\Phi}=\tau_{2} \Phi_{1}^{*} \tau_{2}$ while $\mu_{h}$ as the bare mass term for scalar doublets and $\mu_{1,2}, M^{\prime}$ being the trilinear coupling parameters having mass dimension one. Minimization of this potential with respect to the various fields would give us the consistency conditions and relationships between the various vevs [12. The minimization condition that is relevant to our discussions is given as

$$
\begin{gathered}
v_{R} \frac{\partial V}{\partial v_{L}}-v_{L} \frac{\partial V}{\partial v_{R}}=0 \\
\Rightarrow 2 M^{\prime} \eta_{P} v_{L} v_{R}-\left(\mu_{1} k_{1}+\mu_{2} k_{2}\right)\left(v_{R}^{2}-v_{L}^{2}\right)=0 .
\end{gathered}
$$

From the above expression it is evident that the minimum allows a left-right symmetric solution only for $\eta_{P}=0$. When the $D$-parity is broken by the vev of the $D$-parity odd singlet scalar $\langle\eta\rangle=\eta_{P}$, the vev of $H_{L}$ becomes much smaller than the vev of $H_{R}$. Defining $M_{D}$ as the $D$-parity breaking scale and using allowed model parameters,

$$
\eta_{P} \simeq M^{\prime} \simeq M_{D}, \quad v_{R}>k_{1}, k_{2} \sim M_{W},
$$

we obtain

$$
v_{L} \simeq \frac{-\left(\mu_{1} k_{1}+\mu_{2} k_{2}\right) v_{R}}{M^{\prime} \eta_{P}},
$$

which allows $\eta_{P} \gg v_{R} \gg v_{L}$. The suppression of the vev $v_{L}$ by the $D$-parity breaking scale is what suppresses the neutrino mass. A representative set of parameters would be $\eta_{P}=\mathcal{O}\left(10^{9}\right) \mathrm{GeV}, v_{R}=\mathcal{O}(1) \mathrm{TeV}$ and $v_{L}=$ $\mathcal{O}\left(10^{-9}\right) \mathrm{GeV}$ which gives rise to correct neutrino masses.

Due to the spontaneous $D$-parity breaking, the effective masses of the left-handed and the right-handed doublet scalar fields $H_{L}$ and $H_{R}$ are obtained from Eq. (6) as

$$
\mu_{L}^{2}=\mu_{h}^{2}+M^{\prime} \eta_{P}, \quad \mu_{R}^{2}=\mu_{h}^{2}-M^{\prime} \eta_{P} .
$$

Similarly to the $D$-parity violating conditions in models with triplet Higgs scalars [6], a fine-tuning of the parameter $M$ for a given value of the vev of the singlet field $\eta_{P}$ can allow a right-handed doublet field $H_{R}$ with mass in the $\mathrm{TeV}$ range so as to make it accessible at the LHC. At the same time, the mass of the left-handed scalar doublet $H_{L}$ can be several orders of magnitude larger, which then implies that the vev of this field must be orders of magnitude smaller than the electroweak symmetry breaking scale. In this work we will assume that the Higgs fields are heavier than the right-handed $W_{R}$ boson to be discussed below. This will simplify the discussion of the LHC signatures as the only non-SM particle assumed lighter than $W_{R}$ will be a heavy neutrino.

\section{B. Gauge bosons}

After spontaneous symmetry breaking of the left-right gauge and the $D$-parity the mass matrix for the left- and right-handed charged gauge bosons $W_{L}, W_{R}$ is [12]

$$
M_{W^{ \pm}}^{2}=\frac{1}{4}\left(\begin{array}{c|cc} 
& W_{L}^{+} & W_{R}^{+} \\
\hline W_{L}^{-} & g_{L}^{2}\left(k^{2}+v_{L}^{2}\right) & -2 g_{L} g_{R} k_{1}^{*} k_{2} \\
W_{R}^{-} & -2 g_{L} g_{R} k_{1} k_{2}^{*} & g_{R}^{2}\left(k^{2}+v_{R}^{2}\right)
\end{array}\right),
$$

where $k^{2}=\left|k_{1}\right|^{2}+\left|k_{2}\right|^{2}$. Diagonalizing the above mass matrix, the physical masses of the charged gauge bosons are

$$
\begin{aligned}
M_{W}^{2} & \approx \frac{1}{4} g_{L}^{2}\left(\left|k_{1}\right|^{2}+\left|k_{2}\right|^{2}\right), \\
M_{W_{R}}^{2} & \approx \frac{1}{4} g_{R}^{2} v_{R}^{2},
\end{aligned}
$$

where $g_{L}\left(g_{R}\right)$ is the gauge coupling for gauge group $S U(2)_{L}\left(S U(2)_{R}\right)$. The mixing between the left-handed and right-handed gauge bosons which can be obtained from Eq. 10 is

$$
\left|\sin \theta_{\mathrm{LR}}^{W}\right| \approx 2 \frac{g_{R}}{g_{L}} \frac{M_{W}^{2}}{M_{W_{R}}^{2}} \frac{k_{2} / k_{1}}{1+k_{2}^{2} / k_{1}^{2}} .
$$

Choosing a hierarchy between the vevs of the bi-doublet can suppress this mixing, but a mild hierarchy is phenomenologically consistent with fermion masses with large hierarchy between respective Yukawa couplings. 
With $g_{R} / g_{L} \approx 0.6$ from the embedding in $\mathrm{SO}(10)$ (cf. Section IV) one can get the $W_{L}-W_{R}$ mixing around $10^{-3}$ with a $W_{R}$ mass around $2 \mathrm{TeV}$ as suggested by the diboson excess (cf. Section V).

Similarly, we can write down the mass-squared matrix for the neutral gauge bosons $W_{L}^{3}, W_{R}^{3}$ and $B$ as [12]

$$
M^{2}=\frac{1}{4}\left(\begin{array}{ccc}
g_{L}^{2}\left(k^{2}+v_{L}^{2}\right) & -g_{L} g_{R} k^{2} & -g_{L} g_{B L} v_{L}^{2} \\
-g_{L} g_{R} k^{2} & g_{R}^{2}\left(k^{2}+v_{R}^{2}\right) & -g_{R} g_{B L} v_{R}^{2} \\
-g_{L} g_{B L} v_{L}^{2} & -g_{R} g_{B L} v_{R}^{2} & g_{B L}^{2}\left(v_{L}^{2}+v_{R}^{2}\right)
\end{array}\right)
$$

where $g_{B L}$ is the gauge coupling for $U(1)_{B-L}$ which is related to the $U(1)_{Y}$ SM gauge coupling as $1 / g_{Y}^{2}=$ $1 / g_{R}^{2}+1 / g_{B L}^{2}$. It is clear from the above mass matrix that one of the mass eigenvalues is zero as the determinant vanishes and we denote this physical state as photon. The diagonalization procedure yields SM $Z$ and heavy $Z_{R}$ mass eigenstates

$$
\begin{aligned}
M_{Z}^{2} & \approx \frac{1}{2} \frac{g_{L}^{2}}{c_{W}^{2}}\left(k^{2}+v_{L}^{2}\right)-\frac{g_{L}^{2}}{2 c_{W}^{2} v_{R}^{2}}\left(c_{M}^{2} k^{2}-s_{M}^{2} v_{L}^{2}\right)^{2}, \\
M_{Z_{R}}^{2} & \approx \frac{1}{2}\left(g_{B L}^{2}+g_{R}^{2}\right) v_{R}^{2}+\frac{1}{2}\left(g_{R}^{2} c_{M}^{2} k^{2}+g_{B L}^{2} s_{M}^{2} v_{L}^{2}\right),
\end{aligned}
$$

where $s_{M} \equiv \sin \theta_{M}=g_{B L} / \sqrt{g_{B L}^{2}+g_{R}^{2}}, c_{M}=\sqrt{1-s_{M}^{2}}$. As we have a hierarchy between the different vevs according to $v_{R} \gg k_{1}, k_{2} \gg v_{L}$, one can expand the heavy gauge boson masses in terms of $1 / v_{R}^{2}$ as

$$
\begin{aligned}
M_{W_{R}}^{2} & \approx \frac{1}{2} g_{R}^{2} v_{R}^{2}\left(1+\frac{k^{2}}{v_{R}^{2}}\right) \\
M_{Z_{R}}^{2} & \approx \frac{1}{2}\left(g_{B L}^{2}+g_{R}^{2}\right) v_{R}^{2}\left(1+\frac{c_{M}^{4} k^{2}+s_{M}^{4} v_{L}^{2}}{v_{R}^{2}}\right)
\end{aligned}
$$

For generic $g_{R} \neq g_{L}$ scenarios, the mass relation between the two gauge bosons is given by

$$
\frac{M_{Z_{R}}}{M_{W_{R}}}=\frac{\sqrt{2} g_{R} / g_{L}}{\sqrt{\left(g_{R} / g_{L}\right)^{2}-\tan ^{2} \theta_{W}}}
$$

with the SM weak angle $\theta_{W}$. Hence, $M_{Z_{R}}>M_{W_{R}}$ and numerically for $g_{R} / g_{L}=0.6$ we have $M_{Z_{R}} \approx 4 M_{W_{R}}$. Using the above relation one can derive a lower bound $g_{R} / g_{L}>0.56$. Using $8 \mathrm{TeV}$ ATLAS $20.3 \mathrm{fb}^{-1}$ luminosity data, the derived bound on $Z_{R}$ is $M_{Z_{R}}>2.2 \mathrm{TeV}$ [13] for a range of $g_{R}$ values consistent with the above mass relation. It is interesting to note that the ratio of couplings needed to reconcile the excesses in our model is close to the theoretical limit, making the mass of the right-handed $Z$ boson arbitrarily heavier as compared to $W_{R}$. This means, that the $Z_{R}$ will be potentially out of reach at the $\mathrm{LHC}$.

\section{NEUTRINO MASS VIA LINEAR SEESAW}

Let us write down the relevant Yukawa terms in the Lagrangian that contribute to the fermion masses [12],

$$
\begin{aligned}
\mathcal{L}_{\text {Yuk }} & =h_{\ell} \overline{\ell_{R}} \Phi \ell_{L}+\widetilde{h_{\ell}} \overline{\ell_{R}} \widetilde{\Phi} \ell_{L}+f_{R} \bar{S} \widetilde{H_{R}} \ell_{R} \\
& +f_{L} \bar{S} \widetilde{H_{L}} \ell_{L}+\mu_{S} \overline{\left(S_{L}\right)^{c}} S_{L}+\text { h.c. },
\end{aligned}
$$

with $\widetilde{H_{j}}=i \tau_{2} H_{j}^{*}(j=L, R)$ and $\widetilde{\Phi}=\tau_{2} \Phi^{*} \tau_{2}$. The singlet Majorana field $S$ in Eq. (17) is defined as

$$
S=\left(S_{L}+\left(S_{L}\right)^{c}\right) / \sqrt{2} .
$$

The vevs of $H_{L}, H_{R}$ and $\Phi$ will generate a mass matrix of the neutral fermions in the Majorana basis $\psi_{L}^{T}=$ $\left(\nu_{L},\left(\nu^{c}\right)_{L}, S_{L}\right)$ as

$$
\mathcal{L}_{\text {mass }}=\left(\overline{\left(\nu_{L}\right)^{c}} \bar{N} \overline{\left(S_{L}\right)^{c}}\right) M_{N}\left(\begin{array}{c}
\nu_{L} \\
\left(\nu^{c}\right)_{L} \\
S_{L}
\end{array}\right)
$$

with the neutral lepton mass matrix

$$
\begin{aligned}
& M_{N}=\left(\begin{array}{ccc}
0 & h_{\ell} k_{1}+\tilde{h}_{\ell} k_{2} & f_{L} v_{L} \\
h_{\ell}^{T} k_{1}+\tilde{h}_{\ell}^{T} k_{2} & 0 & f_{R} v_{R} \\
f_{L}^{T} v_{L} & f_{R}^{T} v_{R} & \mu_{S}
\end{array}\right) \\
& \equiv\left(\begin{array}{ccc}
0 & m_{D} & m_{L} \\
m_{D}^{T} & 0 & M \\
m_{L}^{T} & M^{T} & \mu_{S}
\end{array}\right) .
\end{aligned}
$$

From the interaction Lagrangian given in Eq. (17), $m_{D}=$ $h_{\ell} k_{1}+\widetilde{h}_{\ell} k_{2}$ is the Dirac neutrino mass mixing for the leftand right-handed neutrino states, $M=f_{R}\left\langle H_{R}\right\rangle=f_{R} v_{R}$ is the heaviest Dirac neutrino mass term mixing $N-S$ while $m_{L}=f_{L}\left\langle H_{L}\right\rangle=f_{L} v_{L}$ is the small lepton number violating term arising from the induced vev of $H_{L}$.

It is usually difficult to get a small mass $\mu_{S}$ for the singlet field $S$ in any left-right symmetric model, particularly the ones originating from a Grand Unified Theory (GUT). In the present model, we prevent the bare mass of the field $S$ by introducing a global $U(1)_{X}$ symmetry, and assigning $X=1$ for the field $S$ and $X=-1$ for the fields $H_{L}$ and $H_{R}$. This ensures $\mu_{S}=0$ while allowing all other terms in the mass matrix. Although the vev of the field $H_{R}$ breaks the $U(1)_{X}$ global symmetry, this cannot generate $\mu_{S}$ as there are no $D$-parity even singlet scalar fields acquiring vevs in the model. The $D$-parity odd field $\eta$ does not have any interaction with the field $S$, and hence, its vev cannot contribute to the mass of $S$. Therefore, the neutrino mass is entirely given by a linear seesaw. From Eqs. (8), (19) and with the hierarchy $M>m_{D} \gg m_{L}$ we obtain the light neutrino mass matrix as

$$
\begin{aligned}
m_{\nu} & =\frac{\mu_{1} k_{1}+\mu_{2} k_{2}}{M^{\prime} \eta_{P}}\left[m_{D}^{T}\left(f_{R}^{-1} f_{L}\right)+\left(f_{L}^{T} f_{R}^{-1^{T}}\right) m_{D}\right] \\
& =m_{D}^{T} M^{-1} m_{L}+\text { transpose }
\end{aligned}
$$




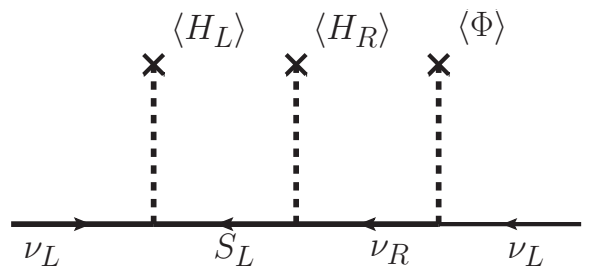

FIG. 1: Majorana mass of the light neutrinos arising through the combined vevs of $H_{L}$ and $H_{R}$.

Additionally, we get two heavy pseudo-Dirac states, whose masses are separated by the light neutrino mass, given by

$$
\widetilde{M} \approx \pm M+m_{\nu}
$$

From Eq. 20 it is clear that the light neutrino mass is suppressed by the parity breaking scale. The smallness of $v_{L}$ thus ensures the smallness of the observed sub-eV scale neutrino masses. The $S U(2)_{R} \times U(1)_{B-L}$ breaking scale $v_{R}$ can be as low as a few TeV. This is in contrast to the usual left-right symmetric model without $D$-parity, where the neutrino mass is suppressed by $v_{R}$ and hence cannot be brought to $\mathrm{TeV}$ scales easily.

Note that the vevs of $H_{L}$ and $H_{R}$ break lepton number by one unit individually; thus, their combination gives the Majorana nature of light neutrinos. This can also be understood from the Feynman diagram as shown in Fig. 1.

\section{A. Unitarity and lepton flavor violation}

Order-of-magnitude-wise and neglecting any flavour structure, we have $m_{D} \sim 1 \mathrm{GeV}, M \sim 10^{3} \mathrm{GeV}$ and $m_{L} \sim 100 \mathrm{eV}$, resulting in $m_{\nu}$ of order $0.1 \mathrm{eV}$. As usual, seesaw mechanisms with additional fermions induce violation of unitarity [14]. Defining $\Theta$, as the mixing between light and heavy neutrinos, and $\epsilon$, as a measure of deviation from unitarity in the PMNS mixing matrix in the light neutrino sector, one can express the leptonic mixing matrix as $N=(1+\epsilon) U^{\mathrm{PMNS}}$ with [15]

$$
\epsilon \simeq-2 m_{D}\left(M^{T}\right)^{-1}\left(M^{*}\right)^{-1} m_{D}^{\dagger} \equiv-2 \Theta \Theta^{\dagger} .
$$

The unitarity violation is therefore of the order $m_{D}^{2} / M^{2}$. The off-diagonal $e \mu, e \tau$ and $\mu \tau$ elements of $\epsilon$ are currently constrained to be smaller than $10^{-5}, 10^{-4}$ and $4 \times 10^{-4}$, respectively [15]. In particular the $e \mu$ entry plays a leading role, as it induces the decay $\mu \rightarrow e \gamma$. In fact, the branching ratio is given by [16]

$$
\mathrm{BR}(\mu \rightarrow e \gamma)=\frac{3 \alpha}{32 \pi} \sum_{i=1}^{3} f\left(\frac{M_{i}}{M_{W}}\right)\left|\Theta_{\mu i}^{*} \Theta_{e i}\right|^{2}
$$

where $M_{i}$ are the heavy neutral fermion masses and $f\left(M_{i}^{2} / M_{W}^{2}\right)$ is a loop-function of order one. We do not study in detail here the flavor structure of the Yukawa matrices, but setting $M_{i}=1 \mathrm{TeV}$ would result in

$$
\mathrm{BR}(\mu \rightarrow e \gamma) \simeq 8.4 \times 10^{-14} \cdot\left(\frac{\left|\left(\Theta \Theta^{\dagger}\right)_{e \mu}\right|}{10^{-5}}\right)^{2}
$$

The current limit derived from MEG experiment is $\mathrm{BR}(\mu \rightarrow e \gamma)<5.7 \times 10^{-13}$ [17] giving a similar limit on $\Theta \sim m_{D} / M$ as the unitarity constraints.

Since we work in a left-right symmetric framework, there are several additional diagrams that contribute to low energy lepton flavor violating processes. In the present doublet version of the left-right symmetric model, the most relevant contribution to $\mu \rightarrow e \gamma$ is from the loop exchange of a heavy right-handed $W_{R}$ boson and neutrino $N$. With respect to the LHC signatures we will assume that there is only one $N$ that dominantly couples to electrons, and that the neutrino generations are aligned with the charged lepton flavors. In this scenario we neglect the $W_{R}$ induced flavor violation.

\section{B. Neutrinoless double beta decay}

LRSMs can give rise to a large number of non-standard contributions [18 23 to neutrinoless double beta decay in addition to the standard light neutrino exchange neutrinos. Regarding the heavy neutrinos, as they form three quasi-Dirac pairs of mass $M$ with essentially negligible splitting of order $m_{\nu}$, i.e. of order $10^{-10} \mathrm{GeV}$, their contributions via left- or right-handed currents are negligible. However, the active neutrinos can couple to the right-handed $W_{R}$ bosons through the LR neutrino mixing of order $\Theta \approx m_{D} / M$. Such contributions are a priori not negligible and potentially interesting. Diagrams exist in which a light neutrino mediates neutrinoless double beta decay by coupling to both the left- and right-handed $W$ boson. These contributions, referred to as $\lambda$ and $\eta$ diagrams [18, are not suppressed by the light Majorana neutrino masses, as no helicity flip is required. The former is suppressed by $\left(W / W_{R}\right)^{2}$ in the amplitude as the one of the Fermi interactions in the standard exchange is replaced by a right-handed current, whereas the latter is suppressed by $\sin \theta_{L R}^{W}$ from a $W_{L}-W_{R}$ mixing. The neutrinoless double beta decay amplitudes for these two contributions are approximately given by [18]

$$
\begin{aligned}
& \mathcal{A}_{\lambda}^{0 \nu} \approx \frac{10^{-2}}{|q|}\left(\frac{g_{R}}{g_{L}}\right)^{2}\left(\frac{M_{W}}{M_{W_{R}}}\right)^{2}\left(U^{\mathrm{PMNS}^{\dagger}} \Theta_{e e}\right. \\
& \mathcal{A}_{\eta}^{0 \nu} \approx \frac{1}{|q|}\left(\frac{g_{R}}{g_{L}}\right) \sin \theta_{L R}^{W}\left(U^{\mathrm{PMNS}} \Theta^{\dagger}\right)_{e e}
\end{aligned}
$$

Here, $|q| \simeq 100 \mathrm{MeV}$ is the virtual neutrino momentum and the factor $10^{-2}$ comes from the nuclear matrix element as normalized with respect to the light neutrino exchange diagram (we assume Germanium as isotope). Defining $\langle\theta\rangle^{0 \nu} \equiv\left|\left(U^{\text {PMNS }} \Theta^{\dagger}\right)_{e e}\right|$, the half-life for neutri- 
noless double beta decay arising from the $\lambda$ and $\eta$ diagrams can be expressed as

$$
\begin{aligned}
\frac{3 \times 10^{25} \mathrm{y}}{T_{\lambda}^{0 \nu}} & \approx\left(\frac{g_{R} / g_{L}}{0.6}\right)^{4}\left(\frac{\langle\theta\rangle^{0 \nu}}{10^{-3.0}}\right)^{2}\left(\frac{2 \mathrm{TeV}}{M_{W_{R}}}\right)^{4} \\
\frac{3 \times 10^{25} \mathrm{y}}{T_{\eta}^{0 \nu}} & \approx\left(\frac{g_{R} / g_{L}}{0.6}\right)^{2}\left(\frac{\langle\theta\rangle^{0 \nu}}{10^{-5.3}}\right)^{2}\left(\frac{\left|\sin \theta_{L R}^{W}\right|}{10^{-3}}\right)^{2} .
\end{aligned}
$$

These predictions can be compared with the current limit $T^{0 \nu} \gtrsim 3 \times 10^{25} \mathrm{y}$ on the half-life of neutrinoless double decay [24 26]. This means that the individual contributions would alone begin to saturate the current experimental limit for $M_{W_{R}} \approx 2 \mathrm{TeV}$ and $\sin \theta_{L R}^{W} \approx 10^{-3}$ as suggested by the LHC excesses (cf. section $\mathrm{V}$ ), as well as the potentially large LR neutrino mixing $\langle\theta\rangle^{0 \nu} \approx 10^{-5}-10^{-3}$ in the linear seesaw. In this conclusion, we neglect possible cancellations between contributions. In the regime suggested by the LHC excesses, the $\eta$ diagram contribution to neutrinoless double beta decay half-life gives the strongest constraint on $\langle\theta\rangle^{0 \nu}$ and it thus sharply restricts the allowed mixing between the light and heavy neutrinos.

\section{EMBEDDING IN $S O(10)$ AND $g_{L} / g_{R}$}

Our framework of a LRSM with doublet and bi-doublet Higgs fields can be embedded in a non-supersymmetric $\mathrm{SO}(10)$ model including gauge coupling unification and TeV scale $W_{R}$ gauge bosons. The breaking scheme, which has a Pati-Salam symmetry as an intermediate step can be as follows 6 ]

$$
S O(10) \stackrel{M_{U}}{\longrightarrow} \mathcal{G}_{224 D} \stackrel{M_{D}}{\longrightarrow} \mathcal{G}_{224} \stackrel{M_{C}}{\longrightarrow} \mathcal{G}_{2213} \stackrel{M_{R}}{\longrightarrow} \mathcal{G}_{S M} \stackrel{M_{\text {Z }}}{\longrightarrow} \mathcal{G}_{13}
$$

The spontaneous symmetry breaking of $S O(10)$ down to low energies is provided by different Higgs multiplets contained in various Higgs representations of $S O(10)$, i.e. $10_{H}, 16_{H}, 54_{H}$ and $210_{H}$. The first stage of symmetry breaking $S O(10) \rightarrow \mathcal{G}_{224 D}$ with $g_{L}=g_{R}$ is achieved by assigning a non-zero vev to 54-dimensional representation of $S O(10)$, i.e., $\langle\rho(1,1,1)\rangle \in 54_{H}$. Since $\rho$ is a $D$-parity even scalar and singlet under the Pati-Salam gauge group, the latter remains intact even after $\rho$ taking its non-zero vev. The second stage of symmetry breaking $\mathcal{G}_{224 D} \rightarrow \mathcal{G}_{224}$ happens when the $D$-parity odd scalar singlet $\langle\eta(1,1,1)\rangle \in 210_{H}$ acquires a non-zero vev leading to $g_{L} \neq g_{R}$. The third stage of symmetry breaking $\mathcal{G}_{224} \rightarrow \mathcal{G}_{2213}$ occurs when $\Sigma(1,1,15) \in 210_{H}$ takes a nonzero vev by breaking $S U(4)_{C}$ down to $S U(3)_{c} \times U(1)_{B-L}$ at the mass scale $M_{C}$. The subsequent step of symmetry breaking $\mathcal{G}_{2213}$ down to the SM gauge group $\mathcal{G}_{213}$ is achieved by assigning a non-zero vev to Higgs doublet $H_{R}$ contained in $16_{H}$. The final stage of symmetry breaking is provided by the SM Higgs doublet contained in $10_{H}$ of $S O(10)$.

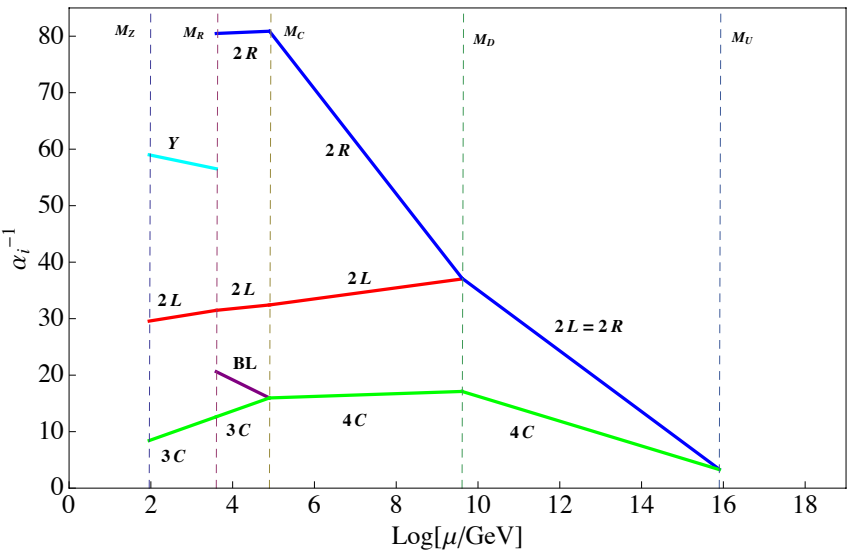

FIG. 2: One loop renormalization group evolution of gauge couplings with Pati-Salam symmetry $G_{224 D}$ as the highest intermediate symmetry breaking.

The motivation for considering such a long chain of symmetry breaking of $S O(10)$ GUT down to the low energy theory $U(1)_{\mathrm{em}} \times S U(3)_{c}$ is as follows: the inclusion of the intermediate symmetry breaking step $\mathcal{G}_{224 D}$ around $10^{8}-10^{10.5} \mathrm{GeV}$ is to give a small vev to $H_{L}$ so that the light neutrino masses can be explained via a linear seesaw mechanism. Moreover, the Pati-Salam symmetry with $D$-parity invariance at the highest intermediate scale ensures gauge coupling unification since above this scale $M_{D}$ ( $D$-parity breaking scale), we have only two gauge couplings to evolve, i.e. $S U(2)_{L} \equiv S U(2)_{R}$ and $S U(4)_{C}$. The inclusion of the subsequent intermediate symmetry breaking $\mathcal{G}_{224}$ at $M_{C}$ is required, as this gives a possibility of explaining the baryon asymmetry of the universe via post-sphaleron baryogenesis [27]. The diquark Higgs scalars, if additionally included in the framework, also get their masses at $M_{C}(\approx$ a few $\mathrm{TeV})$, leading to neutronantineutron oscillation with mixing time close to ongoing experimental search limits $27+29]$. The crucial breaking step $\mathcal{G}_{2213} \rightarrow \mathcal{G}_{S M}$ occurs at the $M_{R}$ scale such that one can have $M_{W_{R}}$ within the TeV range. At the end, the SM symmetry breaking via a bi-doublet is needed for providing correct masses and mixing of fermions as well as to give mass to gauge bosons and the Higgs scalar.

Figure 2 shows the gauge coupling unification, with the associated mass scales and the prediction for the coupling ratio between the $S U(2)_{L}$ and $S U(2)_{R}$ gauge groups near the electroweak scale. However, we have introduced an extra $16_{H^{\prime}}$ and $210_{H^{\prime}}$ in addition to the usual $10_{H}, 16_{H}, 54_{H}$ and $210_{H}$ while performing the renormalization group evolution in order to achieve the unification of gauge couplings. For a detailed discussion on the relevant formalism, see Refs. [22, 29]. From Fig. 2, the various mass scales are found to be $M_{R}=8 \mathrm{TeV}$, $M_{C}=10^{5} \mathrm{GeV}, M_{D}=10^{9.6} \mathrm{GeV}$ and $M_{U}=10^{15.9} \mathrm{GeV}$, which are consistent with the gauge coupling unification while predicting the desired coupling ratio between $g_{L}$ 
and $g_{R}$ at $\mathrm{TeV}$ scale,

$$
\frac{g_{R}}{g_{L}} \approx 0.57 .
$$

This analysis should be understood as a demonstration that our framework can be implemented in GUTs, and as a reminder that left-right symmetric models with $D$ parity breaking allow for different gauge couplings $g_{L} \neq$ $g_{R}$. The latter fact can be used to explain the eejj and dijet excesses at the LHC discussed in the next section. The scenario discussed here is not the only option to achieve differing left and right gauge couplings at the $\mathrm{TeV}$ scale. Other possibilities are for example explored in 30,31 .

\section{LHC SIGNATURES}

\section{A. Experimental picture}

One of the most sensitive processes to probe Left-Right symmetric models at the LHC is given by heavy righthanded $W$ boson and neutrino exchange leading to the signal $p p \rightarrow W_{R} \rightarrow N l_{1}^{ \pm} \rightarrow l_{1}^{ \pm} l_{2}^{ \pm, \mp}+2$ jets [19, 32,38]. Both the CMS and ATLAS collaborations have reported updated bounds on the mass of the right-handed charged gauge boson in the LRSM from their analyses of events at the center of mass energy $\sqrt{s}=8 \mathrm{TeV}$ with an integrated luminosity of $\approx 20 \mathrm{fb}^{-1}$ [7, 39]. The two analyses treat events with two electron and two muons separately but they differ in that the CMS analysis does not differentiate between lepton charges, i.e. it includes both opposite sign and same sign leptons, whereas the ATLAS analysis only considers same sign lepton signatures as expected with a pure Majorana neutrino involved in the process. Observing no significant excess in either the ee, or $\mu \mu$ channel, both collaborations quote a lower limit at $95 \%$ CL on the $W_{R}$ mass of the order $M_{W_{R}}>3 \mathrm{TeV}$, for $M_{N} \approx \frac{1}{2} M_{W_{R}}$.

The CMS data exhibit an excess in the ee channel with a local significance of $2.8 \sigma$ for a $W_{R}$ mass of $M_{W_{R}} \approx 2.1 \mathrm{TeV}$. The excess is seen exclusively in the opposite sign channel $e^{+} e^{-}$, with 13 potential signal events. Only one same sign electron event was observed [7. Neither ATLAS nor CMS attempt to search for lepton flavor violating signatures; instead, the CMS analysis uses $e^{ \pm} \mu^{\mp}$ events to determine the background from data. In the following, we attempt to interpret the CMS excess in our model. This seems especially interesting as there have been a number of additional (individually nonsignificant) excesses around the energy scale $M \approx 2 \mathrm{TeV}$ by both CMS and ATLAS.

When trying to interpret the excess in terms of resonant $W_{R}$ production and decay via an on-shell heavy neutrino, several issues have to be solved:

(i) The eejj excess is too small when compared to the predicted cross section in the minimal LRSM using $g_{R}=$ $g_{L}$ by a factor of $\approx 3-4$. As first reported in [38, this issue is straightforward to understand in LRSMs without manifest left-right symmetry and $g_{R} \approx 0.6 g_{L}$ as is the case in our model.

(ii) Neither ATLAS nor CMS see any excess in same sign lepton channels as expected in LRSMs with a pure Majorana neutrino where the number of same and opposite sign lepton events should be equal. This is easily reconciled in our model which incorporates QuasiDirac heavy neutrinos where the relative mass splitting $\approx m_{\nu} / M$ between the Majorana components is vanishingly small and only opposite sign lepton events are expected.

(iii) There is no excess in the $\mu \mu j j$ channel. In fact CMS sees slightly fewer events than expected from the background for an invariant mass $M_{\mu \mu j j} \approx 1.5 \mathrm{TeV}$. The phenomenologically most straightforward explanation for the lack of the $\mu \mu$ excess is the assumption that only one heavy neutrino is lighter than the $W_{R}$ and it dominantly couples to electrons. This would also mean that the righthanded currents are largely aligned with the charged lepton flavors and the heavy mixing matrix would be close to unity (neglecting the small non-unitarity). We will use this as a working assumption, although other solutions with non-trivial mixing (e.g. to the $\tau$ lepton) are possible [8, 40, 41 ${ }^{1}$.

(iv) The CMS collaboration does not see a localized excess in the invariant mass distribution of the two jets and the sub-leading lepton $l_{2}$ as expected from the decay $N \rightarrow l_{2} j j$. Although the leading and sub-leading lepton cannot be identified on an event-by-event basis, the leptons can be ordered by $p_{T}$, which should produce a characteristic excess distribution in $m_{l_{2} j j}$. It is not clear how significant the absence of such a distribution is as its shape will depend on the mass of the heavy neutrino in the signal process and the low statistics might not allow to draw a conclusion. Another possible explanation is that the signal events are partially generated in another, kinematically different, signal process. An example would be that the neutrino decays in a lefthanded current process via an on-shell SM $W$ boson as discussed below. Alternatively, a second neutrino with a different mass and non-trivially coupling to $\tau$ concurrently contributes to the signal [40]. It is worthwhile to note that the CMS collaboration also does not see localized excesses in other distributions such as based on the invariant masses of other final state particles, e.g. $m_{q q}^{2}$ or $m_{l_{1} l_{2}}^{2}$.

(v) Last but not least, the reported excess of $2.8 \sigma$ lo-

\footnotetext{
${ }^{1}$ By design, the CMS analysis does not report on the potentially possible flavor violating signature $e^{ \pm} \mu^{\mp} j j$ as such events are used to infer the $t \bar{t}$ background. It would be interesting to see if dropping this assumption had any impact; if there were genuine $e^{ \pm} \mu^{\mp} j j$ signal events, one might naively expect that the background is currently overestimated and correcting for this would result in a larger excess and might possibly reduce the underfluctuation of the $\mu \mu j j$ event rate.
} 
cally is currently not high enough to be statistically significant. Nevertheless, the combination with other LHC excesses around the resonant mass $M \approx 2 \mathrm{TeV}$ provides motivation to explore the observed excess despite its insufficient significance. Other analyses in the context of LRSMs were performed in [40, 42,47. The excess has also been discussed in wider theoretical contexts [48 65].

It is indeed intriguing to consider that all the various excesses around $2 \mathrm{TeV}$ can be understood within leftright symmetric scenarios [66, 67]. The different excesses can be summarized as follows:

1. Diboson hadronic final states: Both ATLAS and CMS have performed searches for a resonance hadronically decaying into a pair of the SM gauge bosons 9,68 . The jets emerging from the decays of the gauge bosons are nearly collinear and form a so called fat jet. The ATLAS search has a mild discrimination between fat jets emerging from the decays of $W$ and $Z$. Consequently, the results can be interpreted as decays of a resonance into $W Z$, $Z Z$ and $W W$ final states with overlapping events. ATLAS reports a local excess of $3.4 \sigma, 2.9 \sigma$ and $2.6 \sigma$ in these channels, respectively, in the region $\approx 1.9-2.1 \mathrm{TeV}$, while CMS observes a $1.4 \sigma$ excess at $\approx 1.9 \mathrm{TeV}$ with no discrimination between $W$ and $Z$ tagged jets. It should be noted that there are subtleties involved regarding the jet substructure in the experimental analysis, which could be improved in Run-2 as suggested in e.g. 69.

2. Diboson semileptonic final states: A CMS search [10] for a resonance decaying to SM gauge bosons with leptonically tagged $Z$ sees an excess of $1.5 \sigma$ around $1.8 \mathrm{TeV}$.

3. Gauge boson - Higgs final state: A CMS search [11 for a resonance decaying to SM $W$ and Higgs $H$, where $W$ decays leptonically and a highly boosted $H$ decays to pair of $b$ jets, sees an excess of $2.2 \sigma$ around $1.8-1.9 \mathrm{TeV}$.

4. Dijet final state: Both ATLAS and CMS observe an excess in the dijet distribution of the decay of a resonance to two jets around $1.8 \mathrm{TeV}$ with a significance of $1 \sigma$ and $2.2 \sigma$, respectively [70, 71].

Although the above searches see an excess in the similar mass bins and hence generate a lot of interest, many other searches which are also sensitive to the decays of a $W_{R}$ do not see any excess:

1. Diboson semileptonic final states: ATLAS performed a search for a resonant diboson decay with subsequent leptonic decays of $W$ and hadronic decays of $Z[72$. The search sees no excess of events around $2 \mathrm{TeV}$. ATLAS also performed a search in a diboson final state with leptonically decaying $Z$ and hadronically decaying $W$ [3] with no excess events seen. We do not attempt to explain the nonobservation in this channel in our work. A possible way to reconcile results of this search with other excesses can be found in for example [74.

2. Gauge boson - Higgs final state: Both ATLAS and CMS performed searches for a resonance decaying to a SM gauge boson and a Higgs. While ATLAS searches for leptonic decays of the gauge boson and the Higgs decaying to $b \bar{b}[75$, CMS searches for hadronic decays of the gauge boson and the Higgs decaying to $\tau \tau$ and $W W^{*}$ [76, 77. No excess events were seen in either search.

3. Third generation quarks final states: Any heavy charged particle producing an excess in a dijet final state should also result in signal with third generation SM quarks. Both ATLAS and CMS performed searches for a resonance decaying to top and bottom final states, where the top decays either hadronically [78, or semileptonically [79, 80. Neither of these searches report any excess of events.

\section{B. Theoretical prediction}

Understanding the diboson and dijet results in a common LRSM framework has been attempted the recent work [4]. In order to combine them with an interpretation of the CMS eejj excess, we use the following values for the fitted cross sections derived from the LHC excesses [44,

$$
\begin{aligned}
\sigma\left(p p \rightarrow W_{R} \rightarrow W Z\right) & =5.9_{-3.5}^{+5.3} \mathrm{fb}, \\
\sigma\left(p p \rightarrow W_{R} \rightarrow W H\right) & =4.5_{-4.0}^{+5.2} \mathrm{fb}, \\
\sigma\left(p p \rightarrow W_{R} \rightarrow j j\right) & =91_{-45}^{+53} \mathrm{fb}, \\
\sigma\left(p p \rightarrow W_{R} \rightarrow t b\right) & =0_{-0}^{+39} \mathrm{fb} .
\end{aligned}
$$

The numbers for the individual channels were derived by summing over the bins around 1.8-2.1 TeV and performing a cut and count analysis on this enlarged signal region. The input for producing these numbers are the number of observed events, expected backgrounds, efficiencies and systematic uncertainties, as published by ATLAS and CMS.

In addition to the analysis in [44, we here also include the CMS eejj excess in the analysis. We estimate the cross section $\sigma\left(p p \rightarrow W_{R} \rightarrow N e \rightarrow e e j j\right)$ by taking into account the experimental efficiency $\epsilon=0.754$ for $W_{R} \approx 1.9 \mathrm{TeV}$ and $N_{R} \approx 1.6 \mathrm{TeV}$, cf. table A18 in 7 . The observed number of events is 14 with an expected background of 4 events. We assume all 10 signal events are within the resonance peak and hence derive a signal cross section of $0.66 \mathrm{fb}$. Clearly, the assumption that all signal events belong to the peak is an approximation. We also do not account for any systematic uncertainties or any other factors. However, in order to compensate for this, we assume a rather large error of $0.4 \mathrm{fb}$ on the signal cross section,

$$
\sigma\left(p p \rightarrow W_{R} \rightarrow N e \rightarrow e e j j\right)=0.66_{-0.4}^{+0.4} \mathrm{fb} .
$$


A detailed simulation of various processes is beyond the scope of this paper, but we attempt to demonstrate that the diboson and dijet excesses can be understood together with the CMS eejj excess within the context of LRSMs. The total cross section for $W_{R}^{+}$and $W_{R}^{-}$can be expressed as 83 .

$$
\sigma\left(p p \rightarrow W_{R}\right)=\frac{\pi}{12} \frac{g_{R}^{2}}{s}\left[f_{u \bar{d}}\left(\frac{M_{W_{R}}}{\sqrt{s}}\right)+f_{d \bar{u}}\left(\frac{M_{W_{R}}}{\sqrt{s}}\right)\right] .
$$

The function $f_{q_{1} q_{2}}=A_{q_{1} q_{2}} \exp \left(-B_{q_{1} q_{2}} M_{W_{R}} / \sqrt{s}\right)$ approximates the PDF folded cross section of a resonant production with the fitting parameters in this case given by $A_{u \bar{d}}=2750, B_{u \bar{d}}=37$ and $A_{d \bar{u}}=1065, B_{d \bar{u}}=36$ 84. For $M_{W_{R}}=1.9 \mathrm{TeV}$ and $\sqrt{s}=8 \mathrm{TeV}$, this yields the cross section

$$
\sigma\left(p p \rightarrow W_{R}\right)=390 \mathrm{fb} \cdot\left(\frac{g_{R}}{g_{L}}\right)^{2}
$$

Right-handed $W_{R}$ bosons decay as $W_{R} \rightarrow q \bar{q}, e N, W Z$ and $W H$, the latter two decays through the suppressed $W$ boson mixing angle $\propto \sin ^{2} \theta_{L R}^{W}$. We assume that there is only one heavy neutrino $N$ lighter than $W_{R}$ and there are no other particles below $M_{W_{R}}$. Neglecting SM particle masses kinematically, the respective partial decay widths as enumerated in [44] are given as

$$
\begin{aligned}
\Gamma\left(W_{R}^{+} \rightarrow \sum_{i} q_{i} \bar{q}_{i}\right) & =\frac{3 g_{R}^{2}}{16 \pi} M_{W_{R}}, \\
\Gamma\left(W_{R}^{+} \rightarrow e^{+} N\right) & =\frac{g_{R}^{2} M_{W_{R}}}{48 \pi}\left(1-\frac{M_{N}^{2}}{M_{W_{R}}^{2}}\right)^{2}\left(1+\frac{1}{2} \frac{M_{N}^{2}}{M_{W_{R}}^{2}}\right), \\
\Gamma\left(W_{R}^{+} \rightarrow W^{+} Z\right) & =\frac{g_{L}^{2}}{192 \pi} \sin ^{2} \theta_{L R}^{W} \frac{M_{W_{R}}^{5}}{M_{W}^{4}}, \\
\Gamma\left(W_{R}^{+} \rightarrow W^{+} H\right) & =\frac{g_{L}^{2}}{192 \pi} \sin ^{2} \theta_{L R}^{W} \frac{M_{W_{R}}^{5}}{M_{W}^{4}}
\end{aligned}
$$

The heavy neutrino decays for the eejj signature through the three-body decay $N \rightarrow$ ejj via an off-shell $W_{R}$. Allowing for the potentially sizable $W$ boson $\left(\sin \theta_{L R}^{W}\right)$ and LR neutrino $\left(\sin \theta_{L R}^{N} \equiv \Theta_{e 1}\right.$, i.e. the coupling of the only accessible neutrino to the electron due to LR mixing) mixing, it may also decay as $N \rightarrow e W, \nu_{e} Z$ and $\nu_{e} H$ [85, 86. We assume that the heavy neutrinos are largely aligned with the charged lepton, i.e. the right-handed equivalent of the PMNS mixing matrix is close to unity and $V_{N e} \approx 1$. In our calculations we treat the accessible heavy neutrino as a Dirac particle. Again neglecting SM particle masses kinematically, the respective partial

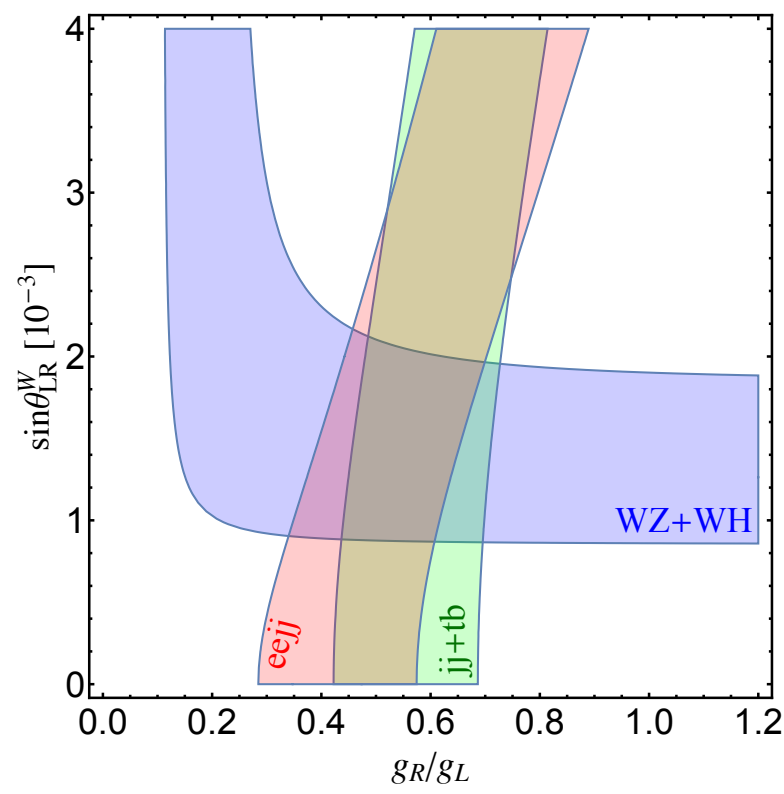

FIG. 3: Fitting the diboson $W Z, W H$ (blue band), dijet $j j+t b$ (green band) and eejj (red band) excesses in the $\left(g_{R} / g_{L^{-}}\right.$ $\left.\sin \theta_{L R}^{W}\right)$ parameter plane. The other parameters are chosen as $M_{W_{R}}=1.9 \mathrm{TeV}, M_{N}=1.6 \mathrm{TeV}$ and $\sin \theta_{L R}^{N}=0$.

decay widths as discussed in [8, 87] are

$$
\begin{aligned}
\Gamma\left(N \rightarrow e^{-} \sum_{i} q_{i} \bar{q}_{i}\right) & =\frac{9 g_{R}^{4}}{2048 \pi^{3}} \frac{M_{N}^{5}}{M_{W_{R}}^{4}}, \\
\Gamma\left(N \rightarrow e^{-} W^{+}\right) & =\frac{g_{L}^{2} \sin ^{2} \theta_{L R}^{N}+g_{R}^{2} \sin ^{2} \theta_{L R}^{W}}{64 \pi} \frac{M_{N}^{3}}{M_{W}^{2}}, \\
\Gamma\left(N \rightarrow \nu_{e} Z\right) & =\frac{g_{L}^{2} \sin ^{2} \theta_{L R}^{N}}{64 \pi \cos ^{2} \theta_{W}} \frac{M_{N}^{3}}{M_{Z}^{2}} \\
\Gamma(N \rightarrow Z H) & =\frac{g_{L}^{2} \sin ^{2} \theta_{L R}^{N}}{64 \pi} \frac{M_{N}^{3}}{M_{H}^{2}}
\end{aligned}
$$

'The above decay widths of $W_{R}$ and $N$ include all relevant couplings, especially the $W-W_{R}$ and $\nu-N$ mixing, consistently, which has not been done in previous analyses of the LHC excesses. Note, although we have given the formulas in the massless SM limit, we use the complete mass dependence in our calculation. It is also important to point out that we assume that the additional scalars in our model are heavier than $W_{R}$ and $N_{R}$, and hence their decays are not present. In case such mass hierarchy is not possible, the decays of $W_{R}, N_{R}$ to the heavy scalars should also be taken into account.

Combining the $W_{R}$ production cross section and the decay widths, we can compare the experimentally suggested cross sections in Eqs. (28) and (29) with the theoretical predictions expressed in terms of the model parameters $g_{R}, \sin \theta_{L R}^{W}, \sin \theta_{L R}^{N}$ and $M_{N}$. We assume that the $W_{R}$ mass is given by $M_{W_{R}}=1.9 \mathrm{TeV}$. Figure 3 shows the compatibility of the theoretically predicted cross sections with the experimentally suggested ranges in the $g_{R} / g_{L}-\sin \theta_{L R}^{W}$ parameter plane. The re- 


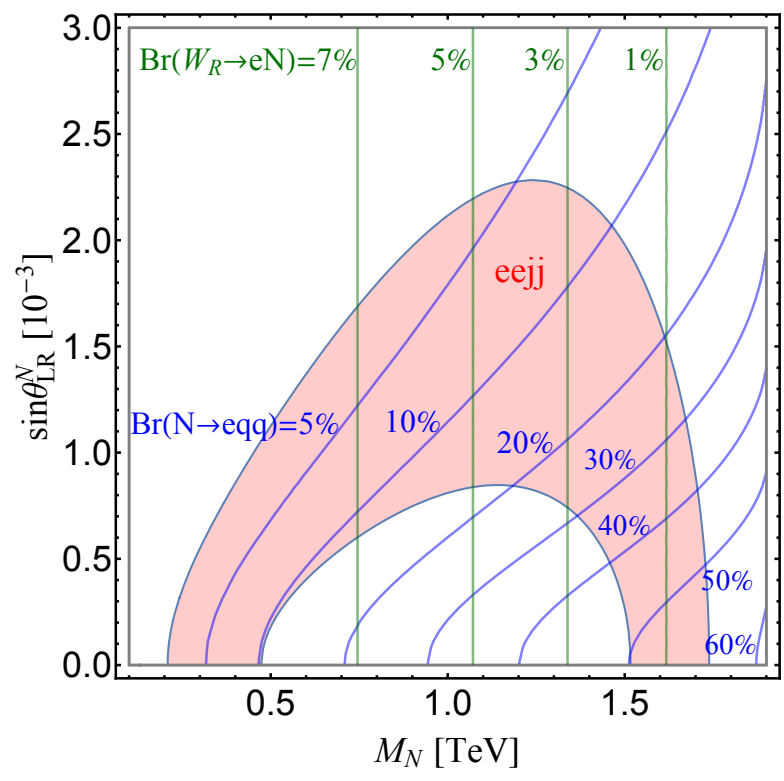

FIG. 4: Fitting the eejj (red band) excess in the $\left(M_{N^{-}}\right.$ $\left.\sin \theta_{L R}^{N}\right)$ parameter plane. The other parameters are chosen as $M_{W_{R}}=1.9 \mathrm{TeV}, g_{R} / g_{L}=0.57$ and $\sin \theta_{L R}^{W}=1.5 \times 10^{-3}$. The vertical green lines denote contours of constant $\operatorname{Br}\left(W_{R} \rightarrow\right.$ $\left.e N_{R}\right)$ and the diagonal blue lines of constant $\operatorname{Br}\left(N_{R} \rightarrow e q q\right)$ as denoted.

maining parameters are chosen as $M_{N}=1.6 \mathrm{TeV}$ and $\sin \theta_{N}=0$. As can be seen and as has been noted in [4], the dijet excess (assuming compatibility with the nonobservation of $t b$ ) points to a right-handed gauge coupling with $g_{R} / g_{L} \approx 0.6$ whereas the diboson excesses suggest a sizeable $W$ boson mixing of $\approx 1.5 \times 10^{-3}$. It is now very interesting to see from our analysis that the eejj excess can be explained for the same values together with the diboson and dijet excesses.

The diboson and dijet cross sections only depend weakly on the neutrino mass and the LR neutrino mixing. On the other hand, the eejj cross section delicately depends on these parameters through the branching ratios $\operatorname{Br}\left(W_{R} \rightarrow e N\right)$ and $\operatorname{Br}(N \rightarrow e q q)$. The latter is a threebody decay and thereby suppressed as $\left(M_{N} / M_{W_{R}}\right)^{4}$. For a small neutrino mass, this decay has to compete with the two-body decays via left-handed current due to the small but sizable LR $W$ boson mixing and the potentially sizable LR neutrino mixing, cf. Eq. (33). The compatibility of the predicted eejj cross section with the observed excess is shown in Fig. 4 as a red band in the $\left(M_{N}-\sin \theta_{L R}^{N}\right)$ parameter plane. The other parameters are fixed to lie in the 'best fit' intersection of Fig. 3. The other excesses are compatible for this choice over the whole $\left(M_{N}-\sin \theta_{L R}^{N}\right)$ parameter plane within uncertainties. Also shown are iso-contours for the branching ratios $\operatorname{Br}\left(W_{R} \rightarrow e N\right)$ and $\operatorname{Br}(N \rightarrow e q q)$, the product of which results in the shape of the red region. For negligible LR neutrino mixing $\sin \theta_{L R}^{N} \approx 0$, there are two solutions: a large neutrino mass $M_{N} \approx 1.6 \mathrm{TeV}$ (as used explicitly in Fig. 3) and a small neutrino mass $M_{N} \approx 0.3 \mathrm{TeV}$. In between these values, the excess could be explained through the inclusion of large LR neutrino mixing up to $\sin \theta_{L R}^{N} \approx 2 \times 10^{-3}$, of the same order as the suggested LR $W$ boson mixing. We thus demonstrate that by interpreting the excesses in such a simplified LRSM would have profound impact on the properties of the heavy neutrino.

It is interesting to note that for such a sizable LR mixing, the heavy neutrino can dominantly decay via two-body left-handed current processes. The left-most blue contour in Fig. 4 denotes a constant branching ratio $\operatorname{Br}(N \rightarrow e q q)=5 \%$, i.e. with the remaining $95 \%$, the neutrino decays via a left-handed current process. This opens up the possibility that the eejj final state is not only produced through $W_{R} \rightarrow e N \rightarrow e e W_{R}^{*} \rightarrow e e j j$ but also $W_{R} \rightarrow e N \rightarrow e e W \rightarrow e e j j$ via a SM $W$. While the kinematics and topology, and thereby the selection efficiency in the respective search, are very much different for the latter process, it may be able to 'pollute' the eejj signal. Although a very tentative conjecture, this may explain the absence of a localized excess in the $m_{e_{2} q q}^{2}$ distribution of the eejj search. This situation is especially expected to arise for lighter heavy neutrino masses, as this scenario favors the neutrino two-body decay and the resulting SM $W$ would not be so strongly boosted, increasing the chance of reconstructing the jets.

\section{CONCLUSIONS}

The excesses around the resonant energy of $2 \mathrm{TeV}$ found in LHC searches are arguably the most promising hint for new physics to emerge at the LHC so far. While individually not very significant due to the large number of LHC searches, their coincidence at around $2 \mathrm{TeV}$ provides motivation to interpret them. In this work we have attempted to do so in a left-right symmetric model where the excesses are produced through the decays of a right-handed $W_{R}$ boson with mass $\approx 2 \mathrm{TeV}$.

The right-handed charged $W_{R}$ boson naturally arises in a left-right symmetric model with Higgs doublets and spontaneous $D$-parity breaking. Unlike manifest leftright symmetric models where the gauge couplings satisfy $g_{R}=g_{L}$, in the present case we have $g_{R} / g_{L} \approx 0.6$ around the $\mathrm{TeV}$ scale. This value is predicted due to $D$-parity breaking in an $S O(10)$ GUT embedding with Pati-Salam symmetry $S U(2)_{L} \times S U(2)_{R} \times S U(4)_{C}$ as its highest subgroup. The sub-eV neutrino masses are explained via a linear seesaw, where the suppression of the neutrino masses come from the high $D$-parity breaking scale. The heavy neutrinos are pseudo-Dirac to a high degree, which means that no signs of lepton number violation would be expected at the LHC. Indeed, 13 of the 14 events in the $p p \rightarrow e e j j$ signal observed by CMS are opposite-sign electrons [7].

Due to the extra suppression of $g_{R}$ in our model, the $W_{R}$ production decreases at the LHC. This helps to understand the eejj and $j j$ excesses as shown in Fig. 3. Interpreting the diboson excesses as decays $W_{R} \rightarrow$ 
$W Z, W H$ fixes the mixing of the $W$ bosons to $\sin \theta_{L R}^{W} \approx$ $10^{-3}$, a value largely compatible with the model prediction $\sin \theta_{L R}^{W} \approx\left(2 g_{R} / g_{L}\right)\left(M_{W} / M_{W_{R}}\right)^{2}$. It is worthwhile to note that the indirect low energy bounds are also ameliorated in our model due to the smaller righthanded gauge coupling. For example, the strongest indirect bound on $M_{W_{R}}$ due to the $K_{L}-K_{S}$ mass difference is roughly given by $\left(g_{R} / g_{L}\right)^{2}\left((2.5 \mathrm{TeV}) / M_{W_{R}}\right)^{2} \lesssim 1$ [88]. In our model, the limit weakens to $M_{W_{R}} \gtrsim 1.5 \mathrm{TeV}$, compatible with the potential signal at $M_{W_{R}} \approx 2 \mathrm{TeV}$ as compared to the limit $M_{W_{R}} \gtrsim 2.5 \mathrm{TeV}$ in manifest LR symmetry.

The main information on the heavy neutrino sector comes from the interpretation of the eejj excess through the decay $W_{R} \rightarrow e N$ where the heavy neutrino $N$ subsequently decays via an off-shell $W_{R}$ as $N \rightarrow$ ejj. We work in a simplified scenario with a single heavy neutrino lighter than $W_{R}$ that has a potentially large mixing $\sin \theta_{L R}^{N} \lesssim 10^{-3}$ with the light neutrinos, cf. Fig. 4 . We would like to highlight that such a large left-right mixing would induce large contributions to neutrinoless double beta decay via the so called $\lambda$ and $\eta$ diagrams. In fact, Eq. (26) show that the limits from neutrinoless double beta decay are of the same order or better for $M_{W_{R}}=2 \mathrm{TeV}$. Especially the $\eta$ contribution results in a stringent constraint on the mixing between the light and heavy neutrinos for $M_{W_{R}} \approx 2 \mathrm{TeV}$ and $\sin \theta_{L R}^{W} \approx 10^{-3}$. Taken at face value, the corresponding limit $\langle\theta\rangle^{0 \nu} \lesssim 10^{-5}$ would restrict the parameter space to the bottom of Fig. 4 with either $M_{N} \approx 0.3 \mathrm{TeV}$ or $M_{N} \approx 1.6 \mathrm{TeV}$. In comparing LHC and neutrinoless double beta decay in this way we assume that the effective mixing parameters are of the same order, $\sin \theta_{L R}^{N} \sim\langle\theta\rangle^{0 \nu}$, which could be violated for non-trivial flavor mixing structures. Large $W$ and neutrino mixing have the effect that at the LHC the purely right-handed current three-body decay of $N$ is competing with left-handed current decays. This can have important implications when interpreting the eejj excess.

We have performed the analysis of the recent excesses, including the CMS eejj excess in an effective LRSM framework that incorporates a single heavy neutrino $N$ lighter than $W_{R}$ and that consistently allows for nonuniversal gauge couplings $g_{R} \neq g_{L}$ and potentially sizable $W-W_{R}$ and $\nu-N$ mixing. Our results, specifically Figs. 3 and 4 apply to other models in such a context.
If the excesses at around $2 \mathrm{TeV}$ were to be confirmed by future data to originate from a $W_{R}$ in a left-right symmetric context, it would have profound implications. The reach of LHC Run-2 for LRSM models for $13 \mathrm{TeV}$ has been explored in a number of papers [40, 44, 81. Due to a rapid increase in the production cross section of $W_{R}$, with a few $\mathrm{fb}^{-1}$ of data, it should be possible to confirm the presence of a $W_{R}$ in the dijet channel. We have assumed that additional scalars in our model are heavier than $M_{W_{R}}$; shall this not be the case, the decays of $W_{R}$ via additional scalars can also be explored at $13 \mathrm{TeV}$. The production of a heavy $Z_{R}$ also present in the model will be difficult because the ratio of gauge couplings is close the theoretically allowed limit and the mass of $Z_{R}$ increases rapidly in this regime. Even disregarding the wider impact on Beyond-the-Standard Model physics and the path of gauge unification, it would directly affect neutrino physics and imply that $B-L$ violation and neutrino mass generation occurs at the TeV scale or below. Moreover, it will strongly disfavor models of high scale leptogenesis 84. In such a case, the explanation of the baryon asymmetry of the universe could be found closely above or even below the electroweak scale [27 29]. Moreover, the $W_{R}$ predicted around $2 \mathrm{TeV}$ can lead to an interesting dark matter phenomenology as explored recently 82 .

\section{Acknowledgements}

The work of SP is partially supported by the Department of Science and Technology, Govt. of India under the financial grant SB/S2/HEP-011/2013 and partly by the Max Planck Society in the project MANITOP. NS is partially supported by the Department of Science and Technology, Govt. of India under the financial grant SR/FTP/PS-209/2011. US is partially supported by the J.C. Bose National Fellowship grant from the Department of Science and Technology, India. The work of WR is supported by the Max Planck Society in the project MANITOP. SK is supported by the 'New Frontiers' program of the Austrian Academy of Sciences. SK thanks J. Tattersall for useful discussions. We thank T. Bandyopadhyay and M. Krauss for pointing out two typos in our paper.
[1] S. Fukuda et al. (Super-Kamiokande), Phys. Rev. Lett. 86, 5656 (2001); Q. R. Ahmad et al. (SNO), Phys. Rev. Lett. 89, 011301 (2002); Phys. Rev. Lett. 89, 011302 (2002); J. N. Bahcall and C. Pena-Garay, New J. Phys. 6, 63 (2004); K. Abe et al. [T2K], Phys. Rev. Lett. 107, 041801 (2011); P. Adamson et al. [MINOS], Phys. Rev. Lett. 107, 181802 (2011); Y. Abe et al. [DOUBLECHOOZ], Phys. Rev. Lett. 108, 131801 (2012); F. P. An et al. [DAYA-BAY], Phys. Rev. Lett. 108, 171803 (2012);
J. K. Ahn et al. [RENO], Phys. Rev. Lett. 108, 191802 (2012).

[2] P. Minkowski, Phys. Lett. B 67, 421 (1977); M. GellMann, P. Ramond and R. Slansky (P. van Niewenhuizen and D. Freedman, eds), (Amsterdam), North Holland, 1979; T. Yanagida, (O. Sawada and A. Sugamoto, eds), (Japan), KEK 1979; R.N. Mohapatra and G. Senjanovic, Phys. Rev. Lett. 44, 912 (1980).

[3] M. Magg and C. Wetterich, Phys. Lett. B 94, 61 (1980); 
T. P. Cheng and L. F. Li, Phys. Rev. D 22 2860, (1980); G. B. Gelmini and M. Roncadelli, Phys. Lett. B 99, 411 (1981); G. Lazarides, Q. Shafi and C. Wetterich, Nucl. Phys. B 181, 287 (1981); R. N. Mohapatra and G. Senjanovic, Phys. Rev. D 23, 165 (1981); J.Schechter and J.W.F. Valle, Phys. ReV. D. 22, 2227 (1980); E. Ma and U. Sarkar, Phys. Rev. Lett. 80, 5716 (1998).

[4] J. C. Pati and A. Salam, Phys. Rev. D10, 275 (1974); R. N. Mohapatra and J. C. Pati, Phys. Rev. D11, 2558 (1975); G. Senjanovic and R. N. Mohapatra, Phys. Rev. D12, 1502 (1975); R. N. Mohapatra and R. E. Marshak, Phys. Rev. Lett. 44, 1316 (1980); N. G. Deshpande, J. F. Gunion, B. Kayser, and F. I. Olness, Phys. Rev. D44, 837 (1991).

[5] M. Malinsky, J.C. Romao and J.W.F. Valle, Phys. Rev. Lett. 95, 161801 (2005); S.M. Barr, Phys. Rev. Lett. 92, 101601 (2004); T. Fkuyama, T.Kikuchi and T. Osaka, J. Cosmol. Astropart. Phys 06 (2005) 005.

[6] D. Chang, R. N. Mohapatra, and M. K. Parida, Phys. Rev. Lett. 52, 1072 (1984); D. Chang, R. N. Mohapatra, and M. K. Parida, Phys. Rev. D 30 (1984) 1052.

[7] V. Khachatryan et al. [CMS Collaboration], Eur. Phys. J. C 74 (2014) 3149, arXiv: 1407.3683 [hep-ex].

[8] J. Gluza and T. Jelinski, arXiv:1504.05568 [hep-ph].

[9] G. Aad et al. [ATLAS Collaboration], arXiv:1506.00962 [hep-ex].

[10] V. Khachatryan et al. [CMS Collaboration], JHEP 1408, 174 (2014) arXiv:1405.3447 [hep-ex]].

[11] CMS Physics Analysis Summary CMS-PAS-EXO-14-010, (2015).

[12] U. Sarkar, Phys. Lett. B 594, 308 (2004) hepph/0403276; N. Sahu and U. Sarkar, Phys. Rev. D 74, 093002 (2006) hep-ph/0605007]; D. Borah, S. Patra and U. Sarkar, Phys. Rev. D 83, 035007 (2011), arXiv:1003.5095 [hep-ph]].

[13] S. Patra, F.S. Queiroz and W. Rodejohann, arXiv:1506.03456 [hep-ph]].

[14] H. Hettmansperger, M. Lindner and W. Rodejohann, JHEP 1104, 123 (2011) arXiv:1102.3432 [hep-ph]].

[15] S. Antusch and O. Fischer, JHEP 1410, 94 (2014) arXiv:1407.6607 [hep-ph]].

[16] A. Ibarra, E. Molinaro and S. T. Petcov, Phys. Rev. D 84, 013005 (2011) arXiv:1103.6217 [hep-ph]].

[17] J. Adam et al. [MEG Collaboration], Phys. Rev. Lett. 110, no. 20, 201801 (2013) arXiv:1303.0754 [hep-ex]].

[18] W. Rodejohann, Int. J. Mod. Phys. E 20, 1833 (2011) arXiv:1106.1334 [hep-ph]]; F. F. Deppisch, M. Hirsch and H. Päs, J. Phys. G 39, 124007 (2012) arXiv:1208.0727 [hep-ph]]. H. Päs and W. Rodejohann, arXiv:1507.00170 [hep-ph].

[19] V. Tello, M. Nemevšek, F. Nesti, G. Senjanović, F. Vissani, Phys. Rev. Lett. 106, 151801 (2011); Joydeep Chakrabortty, H.Zeen Devi, Srubabati Goswami and Sudhanwa Patra, JHEP 1208 (2012) 008; arXiv:1204.2527 [hep-ph].

[20] M.K. Parida and S. Patra, Phys.Lett. B718 (2013) 14071412, arXiv:1211.5000 [hep-ph]].

[21] J. Barry and W. Rodejohann, JHEP 1309, 153 (2013) arXiv:1303.6324 [hep-ph]].

[22] Ram. L. Awasthi, M. K. Parida, and Sudhanwa Patra, JHEP 08, 122 (2013).

[23] D. Stefanik, R. Dvornicky, F. Simkovic and P. Vogel, arXiv:1506.07145 [hep-ph]].

[24] M. Agostini et al., Phys. Rev. Lett. 111 (2013) no. 12,
122503, arXiv:1307.4720.

[25] KamLAND-Zen, A. Gando et al., Phys. Rev. Lett. 110 (2013) no. 6, 062502, arXiv:1211.3863

[26] EXO, M. Auger et al., Search for Neutrinoless DoubleBeta Decay in 136 Xe with EXO-200,? Phys. Rev. Lett. 109 (2012) 032505, arXiv:1205.5608.

[27] K. S. Babu, R. N. Mohapatra and S. Nasri, Phys. Rev. Lett. 97, 131301 (2006) hep-ph/0606144.

[28] K. S. Babu, P. S. Bhupal Dev, E. C. F. S. Fortes and R. N. Mohapatra, Phys. Rev. D 87, no. 11, 115019 (2013) arXiv:1303.6918 [hep-ph]];

[29] S. Patra and P. Pritimita, Eur. Phys. J. C 74, no. 10, 3078 (2014) arXiv:1405.6836 [hep-ph]].

[30] C. Arbelez, M. Hirsch, M. Malinsk and J. C. Romo, Phys. Rev. D 89, no. 3, 035002 (2014) arXiv:1311.3228 [hep$\mathrm{ph}]$.

[31] G. M. Pelaggi, A. Strumia and S. Vignali, arXiv:1507.06848 [hep-ph].

[32] W.-Y. Keung and G. Senjanovic, Phys.Rev.Lett. 50, 1427 (1983).

[33] T.-H. Ho, C.-R. Ching and Z.-J. Tao, Phys.Rev. D42, 2265 (1990).

[34] A. Ferrari et al., Phys. Rev. D62, 013001 (2000).

[35] S. N. Gninenko, M. M. Kirsanov, N. V. Krasnikov and V. A. Matveev, Phys. Atom. Nucl. 70, 441 (2007).

[36] J. A. Aguilar-Saavedra, F. F. Deppisch, O. Kittel and J. W. F. Valle, Phys. Rev. D 85, 091301 (2012).

[37] S. P. Das, F. F. Deppisch, O. Kittel and J. W. F. Valle, Phys. Rev. D 86 (2012) 055006.

[38] F. F. Deppisch, T. E. Gonzalo, S. Patra, N. Sahu and U. Sarkar, Phys. Rev D 90, 053014 (2014); F. F. Deppisch, T. E. Gonzalo, S. Patra, N. Sahu and U. Sarkar, Phys. Rev. D 91, no. 1, 015018 (2015) arXiv:1410.6427 [hep-ph]].

[39] G. Aad et al. [ATLAS Collaboration], arXiv:1506.06020 [hep-ex].

[40] B. A. Dobrescu and Z. Liu, arXiv:1506.06736 [hep-ph].

[41] P. Coloma, B. A. Dobrescu and J. Lopez-Pavon, arXiv:1508.04129 [hep-ph].

[42] M. Heikinheimo, M. Raidal and C. Spethmann, arXiv:1407.6908 [hep-ph].

[43] J. A. Aguilar-Saavedra and F. R. Joaquim, arXiv:1408.2456 [hep-ph].

[44] J. Brehmer, J. Hewett, J. Kopp, T. Rizzo and J. Tattersall, arXiv:1507.00013 [hep-ph].

[45] M. E. Krauss and W. Porod, arXiv:1507.04349 [hep-ph].

[46] K. Cheung, W. Y. Keung, P. Y. Tseng and T. C. Yuan, arXiv:1506.06064 [hep-ph].

[47] M. Dhuria, C. Hati, R. Rangarajan and U. Sarkar, Phys. Rev. D 92, no. 3, 031701 (2015) arXiv:1503.07198 [hep$\mathrm{ph}]]$.

[48] Y. Bai and J. Berger, arXiv:1407.4466 [hep-ph].

[49] S. Biswas, D. Chowdhury, S. Han and S. J. Lee, arXiv:1409.0882 [hep-ph].

[50] B. Allanach, S. Biswas, S. Mondal and M. Mitra, arXiv:1408.5439 [hep-ph].

[51] G. Bambhaniya, J. Chakrabortty, J. Gluza, T. Jelinski and M. Kordiaczynska, arXiv:1408.0774 [hep-ph]; A. Fowlie and L. Marzola, arXiv:1408.6699 [hep-ph]; G. Senjanovic and V. Tello, arXiv:1408.3835 [hep-ph].

[52] G. Cacciapaglia, A. Deandrea and M. Hashimoto, arXiv:1507.03098 [hep-ph].

[53] C. W. Chiang, H. Fukuda, K. Harigaya, M. Ibe and T. T. Yanagida, arXiv:1507.02483 [hep-ph]. 
[54] V. Sanz, arXiv:1507.03553 [hep-ph].

[55] A. Carmona, A. Delgado, M. Quiros and J. Santiago, arXiv:1507.01914 [hep-ph].

[56] B. A. Dobrescu and A. Martin, arXiv:1408.1082 [hep-ph].

[57] L. A. Anchordoqui, I. Antoniadis, H. Goldberg, X. Huang, D. Lust and T. R. Taylor, arXiv:1507.05299 [hep-ph];

[58] J. Hisano, N. Nagata and Y. Omura, arXiv:1506.03931 [hep-ph].

[59] Y. Omura, K. Tobe and K. Tsumura, arXiv:1507.05028 [hep-ph].

[60] S. Fichet and G. von Gersdorff, arXiv:1508.04814 [hep$\mathrm{ph}]$.

[61] A. Thamm, R. Torre and A. Wulzer, arXiv:1506.08688 [hep-ph].

[62] C. Petersson and R. Torre, arXiv:1508.05632 [hep-ph].

[63] Y. Gao, T. Ghosh, K. Sinha and J. H. Yu, arXiv:1506.07511 [hep-ph].

[64] M. Dhuria, C. Hati, R. Rangarajan and U. Sarkar, Phys. Rev. D 91, no. 5, 055010 (2015) arXiv:1501.04815 [hep$\mathrm{ph}]$.

[65] M. Dhuria, C. Hati and U. Sarkar, arXiv:1507.08297 [hep-ph].

[66] F. F. Deppisch, Talk at Nu@Fermilab workshop, July 2015, https://indico.fnal.gov/ contributionDisplay.py? contribId=0\&conf Id=9771 (retrieved on 11 August 2015).

[67] P. S. B. Dev and R. N. Mohapatra, arXiv:1508.02277 [hep-ph].

[68] V. Khachatryan et al. [CMS Collaboration], JHEP 1408, 173 (2014) arXiv:1405.1994 [hep-ex]].

[69] D. Goncalves, F. Krauss and M. Spannowsky, arXiv:1508.04162 [hep-ph].

[70] G. Aad et al. [ATLAS Collaboration], Phys. Rev. D 91, no. 5, 052007 (2015) arXiv:1407.1376 [hep-ex]].

[71] V. Khachatryan et al. [CMS Collaboration], Phys. Rev. D 91, no. 5, 052009 (2015) arXiv:1501.04198 [hep-ex]].

[72] G. Aad et al. [ATLAS Collaboration], Eur. Phys. J. C 75, no. 5, 209 (2015) arXiv:1503.04677 [hep-ex]].

[73] G. Aad et al. [ATLAS Collaboration], Eur. Phys. J. C 75, no. 2, 69 (2015) arXiv:1409.6190 [hep-ex]].
[74] J. A. Aguilar-Saavedra, arXiv:1506.06739 [hep-ph].

[75] G. Aad et al. [ATLAS Collaboration], Eur. Phys. J. C 75, no. 6, 263 (2015) arXiv:1503.08089 [hep-ex]].

[76] V. Khachatryan et al. [CMS Collaboration], Phys. Lett. B 748, 255 (2015) arXiv:1502.04994 [hep-ex]].

[77] V. Khachatryan et al. [CMS Collaboration], arXiv:1506.01443 [hep-ex].

[78] G. Aad et al. [ATLAS Collaboration], Eur. Phys. J. C 75, no. 4, 165 (2015) arXiv:1408.0886 [hep-ex]].

[79] G. Aad et al. [ATLAS Collaboration], Phys. Lett. B 743, 235 (2015) arXiv:1410.4103 [hep-ex]].

[80] S. Chatrchyan et al. [CMS Collaboration], JHEP 1405, 108 (2014) arXiv:1402.2176 [hep-ex]].

[81] B. A. Dobrescu and Z. Liu, arXiv:1507.01923 [hep-ph].

[82] Julian Heeck and Sudhanwa Patra, arXiv:1507.01584 [hep-ph]

[83] A. Leike, Phys. Rept. 317 (1999) 143 hep-ph/9805494.

[84] S. Carlier, J. M. Frere and F. S. Ling, Phys. Rev. D 60, 096003 (1999); J. -M. Frere, T. Hambye and G. Vertongen, JHEP 0901, 051 (2009); F. F. Deppisch, J. Harz and M. Hirsch, Phys. Rev. Lett. 112, 221601 (2014); F. F. Deppisch, J. Harz, M. Hirsch, W. C. Huang and H. Ps, Phys. Rev. D 92, 036005 (2015). P. S. B. Dev, C. H. Lee and R. N. Mohapatra, arXiv:1408.2820 [hepph]; M. Dhuria, C. Hati, R. Rangarajan and U. Sarkar, arXiv:1502.01695 [hep-ph].

[85] C. H. Lee, P. S. Bhupal Dev and R. N. Mohapatra, Phys. Rev. D 88, no. 9, 093010 (2013) arXiv:1309.0774 [hep$\mathrm{ph}]$.

[86] F. F. Deppisch, P. S. Bhupal Dev and A. Pilaftsis, New J. Phys. 17, no. 7, 075019 (2015).

[87] F. del Aguila and J. A. Aguilar-Saavedra, Nucl. Phys. B 813, 22 (2009) arXiv:0808.2468 [hep-ph]].

[88] G. Beall, M. Bander, and A. Soni, Phys. Rev.Lett. 48 (1982) 848; Y. Zhang, H. An, X. Ji and R. N. Mohapatra, Nucl. Phys. B 802, 247 (2008); A. Maiezza, M. Nemevsêk, F. Nesti, and G. Senjanović, Phys. Rev. D 82 (2010) 055022; S. Bertolini, A. Maiezza and F. Nesti, Phys. Rev. D 89, 095028 (2014) arXiv:1403.7112 [hep$\mathrm{ph}]$. 